\title{
Principio de proporcionalidad y sanciones administrativas en la jurisprudencia constitucional
}

\section{Principle of proportionality and administrative sanctions on constitutional case law}

\author{
JOSÉ ANTONIO TIRADO BARRERA*
}

Resumen: El presente trabajo tiene por finalidad mostrar una visión panorámica del tratamiento del principio de proporcionalidad en el ejercicio de la potestad sancionadora de la administración pública por parte de la jurisprudencia del Tribunal Constitucional.

Palabras clave: Sanciones administrativas - principio de proporcionalidad jurisprudencia constitucional - administración pública

Summary: This work aims to show a panoramic perspective of proportionality principle treatment in Public Administration performing power to impose sanctions by Constitutional Court case law.

Key words: Administrative sanctions - principle of proportionality - constitutional case law - Public Administration

CONTENIDO: I. CUESTIONES PRELIMINARES. - II. UNA MIRADA PANORÁMICA A LA JURISPRUDENCIA CONSTITUCIONAL SOBRE SANCIONES ADMINISTRATIVAS Y PROPORCIONALIDAD. - III. A MODO DE CONCLUSIÓN.

\section{CUESTIONES PRELIMINARES}

El presente trabajo tiene por finalidad mostrar una visión panorámica del tratamiento del principio de proporcionalidad en el ejercicio de la potestad sancionadora de la administración pública por parte de la jurisprudencia del Tribunal Constitucional ${ }^{1}$.

Qué duda cabe de que el principio de proporcionalidad constituye uno de los criterios orientadores fundamentales del constitucionalismo moderno y cuyo extraordinario desarrollo ha merecido un tratamiento doctrinario especialmente relevante y una acogida jurisprudencial que no ha sido menor ${ }^{2}$.

* Abogado egresado de la Pontificia Universidad Católica del Perú. Correo electrónico: jtirado @ pucp. edu.pe.

1 Cresci Vassallo, Giancarlo. «El principio de proporcionalidad en el Derecho administrativo sancionador y la jurisprudencia constitucional». Gaceta del Tribunal Constitucional, número 3, julio septiembre 2006.

2 Bernal PULIDO, Carlos. El principio de proporcionalidad y los derechos fundamentales. El principio de proporcionalidad como criterio para determinar el contenido de los derechos fundamentales 
En este sentido, constituye un dato muy relevante encontrar en el Derecho administrativo a algunos de los referentes iniciales de lo que modernamente vendría a configurar el contenido del principio de proporcionalidad $^{3}$, y en la potestad sancionadora de la administración a uno de aquellos espacios más importantes para su aplicación práctica en la defensa y protección de los derechos fundamentales.

De este modo, resulta claro advertir cómo el creciente proceso de constitucionalización, la exigencia de la vigencia práctica de los derechos fundamentales y la racionalización en el ejercicio del poder, hacen que el principio de proporcionalidad y el Derecho administrativo se encuentran íntimamente relacionados, pues aquel se constituye como el instrumento técnico más idóneo para asegurar que: «[...] el ejercicio de los derechos fundamentales no puede ser restringido más allá de lo estrictamente necesario para la tutela de los intereses públicos.» .

Ahora bien, desde el punto de vista de su aplicación, el principio de proporcionalidad en el ámbito del ámbito del Derecho administrativo sancionador se presenta en dos planos claramente diferenciados. En primer lugar, su aplicación en el plano normativo, y, en segundo lugar, en el plano aplicativo ${ }^{5}$. Esto hace que se requieran distintos grados o intensidades en la utilización de los criterios que componen el juicio de proporcionalidad, de acuerdo al ámbito específico en que deba ser aplicado 6 .

Así, debe tenerse presente que, si bien es cierto, el principio de proporcionalidad puede aplicarse tanto respecto de leyes como de actos administrativos, el juzgador que lo aplique deberá tener presente también las diferencias cualitativas entre estos tipos de actuaciones estatales sujetas a fiscalización jurisdiccional ${ }^{7}$, pues si el juez debe ser deferente respecto

vinculantes para el legislador. Segunda edición actualizada. Madrid: Centro de Estudios Políticos y Constitucionales, 2005, p. 873.

3 «El concepto de proporcionalidad nacido en el Derecho Penal, pasa al Derecho de Policía en su etapa de concepción liberal, correspondiente al siglo XIX, que se caracteriza -frente a la concepción absolutista de la policía- por el robustecimiento de la protección de las esferas jurídicas individuales mediante el reconocimiento constitucional de unos derechos que limitan el ejercicio de la potestad policial. En consecuencia, el poder de policía no se presenta ya como un poder general e indeterminado, capaz de instrumentar en su favor cualquier medida interventora escogida por la autoridad, sino por el contrario como un poder limitado por las normas que lo reconocen, por los derechos individuales constitucionalmente garantizados, y en todo caso controlable por los jueces» (LÓPEZ GONZÁLEZ, José Ignacio. El principio general de proporcionalidad en Derecho administrativo. Sevilla: Ediciones del Instituto García Oviedo, 1988, p. 16).

4 lbíd., p. 30.

5 «[...] el principio opera en dos planos: en el normativo, de tal manera que las disposiciones generales han de cuidarse de que las sanciones que asignen a las infracciones sean proporcionales a éstas; $y$ en el de la aplicación, de tal manera que las sanciones singulares que se impongan sean igualmente proporcionales a las infracciones concretas imputadas" (NIETO GARCíA, Alejandro. Derecho administrativo sancionador. Cuarta edición totalmente revisada. Madrid: Tecnos, 2005, p. 351).

6 Una opinión discrepante respecto de la aplicación del principio de proporcionalidad en el ámbito de las sanciones administrativas es la expuesta en: SARMIENTO RAMIREZ-ESCUDERO, Daniel. El control de proporcionalidad de la actividad administrativa. Valencia: Tirant lo Blanch, 2004, p. 258.

7 «[...] la legalidad material de un acto administrativo se fiscaliza en un primer plano con respecto a la conformidad con las leyes y los principios de derecho; en un segundo plano, en cuanto a la conformidad del acto con la reserva de ley; en un tercer plano, con respecto al ejercicio correcto de la potestad discrecional; y en un cuarto plano, bajo los aspectos de la proporcionalidad. No 
de la especial legitimidad democrática del legislador, el control sobre la actuación de la administración puede —y debe — ser mucho más estricto, dado que la administración no puede invocar dicha legitimidad, y porque, además, actúa sometida, precisamente, a los mandatos del legislador democrático .

Ahora bien, antes de avanzar conviene detenernos en una cuestión aparentemente solo terminológica, pero que resulta esencial: ihablamos del principio de proporcionalidad o del principio de razonabilidad? Planteamos esta pregunta porque el Tribunal Constitucional ha utilizado dichos términos en forma de sinónimos ${ }^{9}$ y porque la Ley del Procedimiento Administrativo General, aunque se refiere al principio de razonabilidad, lo define —como veremos más adelante- a partir de los elementos configuradores del principio de proporcionalidad ${ }^{10}$. Corresponde,

obstante, estas aproximaciones similares a la hora de fiscalizar una ley, por un lado, y un acto administrativo, por otro, conviene destacar que en el control de la proporcionalidad de una ley el Tribunal Constitucional alemán respeta siempre el margen de apreciación del legislador, que es, sin excepción, más amplio que aquel de la Administración Pública» (BLANKE, Hermann-Josef. «El principio de proporcionalidad en el Derecho alemán, europeo y latinoamericano". Revista de Derecho Administrativo, año 5, número 9, p. 344, Lima.

8 La aplicación del principio de proporcionalidad en el plano normativo como en el plano aplicativo merece una precisión en el caso del Derecho administrativo peruano. Como regla general, la autoridad administrativa solo podrá utilizar el principio de proporcionalidad en aquellos casos en los que se encuentre habilitada legalmente para la emisión de normas reglamentarias o para el caso de decisiones concretas a través de la emisión de un acto administrativo, salvo que se encuentre dentro de los alcances del precedente vinculante emitido por el Tribunal Constitucional y pueda realizar el denominado control difuso administrativo, en cuya virtud algunos órganos administrativos pueden, expresamente, no aplicar leyes que resulten manifiestamente inconstitucionales. Una opinión en contra de lo sostenido por el Tribunal Constitucional en esta materia ha sido expuesta en nuestro trabajo El control difuso de la constitucionalidad de las leyes por parte de la administración pública en la jurisprudencia del Tribunal Constitucional. Lima: Grijley, 2008, p. 159.

9 «El principio de razonabilidad o proporcionalidad es consustancial al Estado Social y Democrático de Derecho, y está configurado en la Constitución en sus artículos $3^{\circ}$ y $43^{\circ}$, y plasmado expresamente en su artículo $200^{\circ}$, último párrafo. Si bien la doctrina suele hacer distinciones entre el principio de proporcionalidad y el principio de razonabilidad, como estrategias para resolver conflictos de principios constitucionales y orientar al juzgador hacia una decisión que no sea arbitraria sino justa; puede establecerse, prima facie, una similitud entre ambos principios, en la medida que una decisión que se adopta en el marco de convergencia de dos principios constitucionales, cuando no respeta el principio de proporcionalidad, no será razonable. En este sentido, el principio de razonabilidad parece sugerir una valoración respecto del resultado del razonamiento del juzgador expresado en su decisión, mientras que el procedimiento para llegar a este resultado sería la aplicación del principio de proporcionalidad con sus tres subprincipios: de adecuación, de necesidad y de proporcionalidad en sentido estricto o ponderación» (Tribunal Constitucional: expediente 2192-2004-AA/TC).

10 La Ley del Procedimiento Administrativo General (ley 27444) nos dice:

«Título Preliminar.

Artículo IV.- Principios del procedimiento administrativo.

El procedimiento administrativo se sustenta fundamentalmente en los siguientes principios, sin perjuicio de la vigencia de otros principios generales del Derecho Administrativo: [...] Razonabilidad. Las decisiones de la autoridad administrativa, cuando creen obligaciones, califiquen infracciones, impongan sanciones, o establezcan restricciones a los administrados, deben adaptarse dentro de los límites de la facultad atribuida y manteniendo la debida proporción entre los medios a emplear y los fines públicos que deba tutelar, a fin de que respondan a lo estrictamente necesario para la satisfacción de su cometido. [...] Artículo 230.- Principios de la potestad sancionadora administrativa La potestad sancionadora de todas las entidades está regida adicionalmente por los siguientes principios esenciales [...] Razonabilidad. Las autoridades deben prever que la comisión de la conducta sancionable no resulte más ventajosa para el infractor que cumplir las normas infringidas o asumir la sanción. Sin embargo, las sanciones a ser aplicadas deberán ser proporcionales al incumplimiento calificado como infracción, debiendo observar los siguientes criterios que en orden de prelación se señalan a efectos de su graduación: La gravedad del daño al interés público y/o bien jurídico protegido. El perjuicio económico causado. La repetición y/o continuidad en la comisión de la

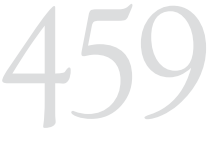

PRINCIPIO DE

PROPOR-

CIONALIDADY

SANCIONES

ADMINISTRATIVAS

EN LA

JURISPRUDENCIA

CONSTITUCIONAL

PRINCIPLE OF

PROPORTIONALITY

AND ADMINISTRATI-

VE SANCTIONS ON CONSTITUTIONAL

CASE LAW 
entonces, preguntarse acerca del contenido de ambos, si es que se trata de conceptos distintos ${ }^{11}$.

Esta cuestión es, como podrá advertirse, una cuestión de gran calado, y que en nuestro medio ha intentado ser resulta a partir del esfuerzo por determinar el contenido de los principios de razonabilidad y de proporcionalidad recogidos en el último párrafo del artículo 200 de la Constitución - aquel que se refiere a los procesos constitucionales en estados de excepción - a partir del criterio de unidad de la esta y de coherencia o concordancia práctica ${ }^{12}$.

Así, se ha señalado que:

Teniendo en cuenta que la razonabilidad implica evaluar una determinada medida desde el punto de vista de su justificación racional, consideramos que debe ser entendida como un paso previo al análisis de proporcionalidad, que consista en verificar que toda medida que limite o restrinja la libertad o los derechos fundamentales, se encuentre justificada en la consecución de un fin legítimo. En tal sentido, la razonabilidad permitiría rechazar todas aquellas medidas que carezcan totalmente de explicación, que sean manifiestamente absurdas o que se justifiquen en la búsqueda de objetivos proscritos por nuestro texto constitucional, de manera explícita o implícita [...] De esta manera, solo una vez superado el tamiz impuesto por este primer principio, sería posible realizar el análisis de proporcionalidad de la medida, evaluación esta última que ya no recaería sobre la medida en sí misma, sino sobre la relación existente entre ésta y el fin que pretende alcanzar, la cual sería efectuada sobre la bases de las exigencias de idoneidad, necesidad y proporcionalidad stricto sensu, que integran este segundo parámetro de constitucionalidad. ${ }^{13}$

A pesar de los fundamentos expuestos, consideramos que puede resultar un tanto forzado darle un contenido diferente al principio de razonabilidad respecto del principio de proporcionalidad y, específicamente, respecto del subprincipio de adecuación, que es el primer componente de aquel.

Si bien es cierto que la Constitución habla de la razonabilidad y de la proporcional del acto restrictivo, de ello no se deriva - necesariamente-

infracción. Las circunstancias de la comisión de la infracción. El beneficio ilegítimamente obtenido; y La existencia o no de intencionalidad en la conducta del infractor".

11 Desde nuestra doctrina también se ha asimilado el concepto de razonabilidad con el de proporcionalidad en el ámbito administrativo. Así, se ha señalado que: «a nuestro entender, compartiendo la posición del Tribunal Constitucional nacional en esta materia, en materia sancionadora administrativa existe similitud entre la proporcionalidad y la razonabilidad, que como principios convergen en enfrentar la arbitrariedad a través de la razón, siendo que la proporcionalidad es la medida de lo razonable de la sanción». (MORÓN URBINA, Juan Carlos. «Los principios de la potestad sancionadora de la administración pública a través de la jurisprudencia del Tribunal Constitucional». Palestra del Tribunal Constitucional. Revista Mensual de Jurisprudencia, año 1. número 7, julio 2006, p. 642).

12 INDACOCHEA PREVOST, Úrsula. «¿Razonabilidad, proporcionalidad o ambos? Una propuesta de delimitación de sus contenidos a partir del concepto de ponderación». En Thēmis. Revista de Derecho, número 55, p. 99.

13 lbíd., pp. 107-108. 
que debamos hablar de principios distintos y que debamos argumentar respecto de la forma en que, siendo distintos, puedan integrarse armónicamente. Ello, básicamente, porque el contenido otorgado a la razonabilidad se integra exactamente en el contenido del subprincipio de adecuación.

Así, mientras que: «la razonabilidad implica evaluar si las restricciones que se imponen a los derechos o a la libertad de los individuos se adecuan a las necesidades y fines públicos que los justifican, de manera que no aparezcan como injustificadas o arbitrarias, sino como razonables; esto es, proporcionadas a las circunstancias que las originan y a los fines que se quiere alcanzar con ellas» ${ }^{14}$, el subprincipio de adecuación o idoneidad establece que: «[...] toda intervención en los derechos fundamentales debe ser adecuada para contribuir a la obtención de un fin constitucionalmente legítimo [...]», y, a su vez, «el subprincipio de idoneidad impone dos exigencias a toda medida de intervención en los derechos fundamentales: en primer lugar, que tenga un fin constitucionalmente legítimo y, en segundo término, que sea idónea para favorecer su obtención» ${ }^{15}$.

Como puede apreciarse, ambos conceptos comparten como un elemento esencial de su configuración la relación existente entre una decisión con capacidad de incidir en un derecho fundamental y la posibilidad de satisfacer - a través de dicha decisión- las exigencias derivadas de otros derechos o bienes constitucionales ${ }^{16}$. En el caso del principio de proporcionalidad, el juicio de adecuación constituye el criterio más deferente respecto del legislador democrático, en la medida en que bastaría que la acción adoptada sea, al menos, adecuada o potencialmente adecuada para entender que se ha superado el juicio de adecuación y pasar a los siguientes niveles de control sobre su necesidad y sobre su proporcionalidad en sentido estricto.

14 lbíd., p. 103.

15 Bernal Pulido, Carlos. Ob. cit., p. 689. Por su parte, Laura Clericó señala que el mandato de adecuación técnica — como también se le conoce al subprincipio de adecuación- «[...] exige la implementación de un medio cuyos efectos contribuyan al fomento del fin perseguido. Visto desde la perspectiva de quien aplica el derecho (en principio, el juez, el administrador) se trata de un control posterior a la selección del medio escogido. Así, se examina si el medio escogido es capaz de fomentar el fin buscado. El mandato de adecuación técnica supone una relación de medio-fin: un medio es escogido e implementado para fomentar un fin (o fines) determinado/s. Esta relación medio-fin presenta igual estructura para el que decide como para el que la controla. Lo que difiere es la perspectiva desde la que se la considera. El legislador mira la relación entre el medio y el fin dese el presente hacia el futuro, confía en que el medio escogido fomenta el logro del fin perseguido, toda vez que primero determina el fin, luego prueba los medios en tanto y en cuanto se relacionan con el fin y supuestamente escoge uno entre los que fomentan el fin. Quien controla, en cambio, prueba a posteriori la relación medio-fin. Comprueba en qué medida el fin perseguido, estado de cosas que se buscó provocar a través de la medida estatal, difiere de (o coincide con) el estado de cosas logrado a través de la implementación del medio escogido por el decisor. En este sentido, el examen de la adecuación técnica se refiere a la relación fáctica entre el medio y el fin» (CLERICó, Laura. El examen de proporcionalidad en el Derecho constitucional. Buenos Aires: Universidad de Buenos Aires, 2009, p. 43).

16 Lo que ha sido reconocido por el propio Tribunal Constitucional cuando señala que: «[...] el principio de proporcionalidad ya lleva consigo, como presupuesto, la exigencia de razonabilidad y, por otra parte, integra adicionalmente el principio de proporcionalidad en sentido estricto o ponderación» (Tribunal Constitucional: expediente 045-2004-Al/TC). 
Por otro lado, el desarrollo teórico del principio de proporcionalidad nos parece mucho más preciso ${ }^{17}$, y esa precisión está orientada al respeto por la legitimidad democrática del legislador y su amplio margen de apreciación en la configuración de los derechos fundamentales, pues a través de la formulación de los subprincipios que lo integran —así como mediante la fijación de un conjunto particularmente importante de reglas interpretativas - se permite superar largamente las críticas sobre su indeterminación o inconmensurabilidad —críticas ya planteadas, precisamente, en su contra-.

De este modo, pues, consideramos que, al menos preliminarmente, es más consistente con la Constitución hablar del principio de proporcionalidad y subsumir en el subprincipio de adecuación los alcances atribuidos al principio de razonabilidad ${ }^{18}$.

\section{UNA MIRADA PANORÁMICA A LA JURISPRUDENCIA CONSTITUCIONAL SOBRE SANCIONES ADMINISTRATIVAS Y PROPORCIONALIDAD}

La revisión de las decisiones adoptadas por el Tribunal Constitucional en relación con la aplicación del principio de proporcionalidad de las sanciones administrativas debe tener presente, entre otros criterios, el de la consagración normativa de este a través de la Ley del Procedimiento Administrativo General en el año 2001. Así, antes de la vigencia formal de la ley 27444, el principio de proporcionalidad de las sanciones administrativas no tenía, en sentido estricto, un reconocimiento positivo que facilitara a los jueces su aplicación o que permitiera a los operadores administrativos su debida observancia. Ciertamente, tal omisión no debería constituir una excusa para permitir actuaciones desproporcionadas, pero, sin duda alguna, su reconocimiento normativo contribuye enormemente a su debida aplicación.

Así, podemos observar que, en una etapa muy inicial de la andadura de nuestro Tribunal Constitucional, las referencias al principio de proporcionalidad son, apenas, una mera cita de su existencia, sin que se haya preocupado el Tribunal por definir su fundamento normativo

17 Por el contrario, se ha dicho que el principio de razonabilidad: «[...] se utiliza por los jueces - señaladamente por el Tribunal Constitucional- para fundamentar sus decisiones y se emplea por la comunidad jurídica y política para valorar la corrección de las decisiones jurisprudenciales. Sin embargo, el concepto de razonabilidad se erige como un concepto ambiguo. Las razones de esta ambigüedad residen en el hecho de que tanto la doctrina como la jurisprudencia del Tribunal Constitucional adscriben múltiples significados a este criterio. De esta manera, no puede hablarse de un significado unívoco y constante del concepto de razonabilidad" (BERNAL PULIDO, Carlos. EI Derecho de los derechos. Escritos sobre la aplicación de los derechos fundamentales. Bogotá: Universidad Externado de Colombia, 2005, p. 68).

18 Cabe señalar que algunos autores resaltan la sustancial identidad de ambos principios. Se recomienda ver CIANCIARDO, Juan. El principio de razonabilidad: del debido proceso sustantivo al moderno principio de proporcionalidad. Buenos Aires: Editorial Abaco de Rodolfo de Palma, 2004, pp. 23 y siguientes. 
ni establecer, con los límites que correspondan, su contenido de forma clara y precisa.

Un ejemplo de esta conducta, que puede ser calificada como intuicionis$\mathrm{ta}^{19}$, es la forma en que el Tribunal aborda el cuestionamiento realizado contra lo dispuesto en el decreto supremo 017-96-PCM.

En esta norma se establecía la prohibición absoluta de reingreso a la función pública de todo servidor cesado por causal de excedencia en el marco del decreto ley 26093. En una de las primeras oportunidades en que el Tribunal Constitucional se refiere a los límites de la potestad sancionadora de la administración pública ${ }^{20}$, señala que una disposición como la reseñada:

[...] no resulta [...] razonable ni proporcional la sanción tan drástica establecida en el Artículo $5^{\circ}$ del Decreto Supremo N ${ }^{\circ}$ 017-96-PCM que impide al servidor público declarado excedente volver a ingresar a laborar en la Administración Pública, siempre que haya puesto vacante, autorización legal para cubrirlo y haya sido evaluado favorablemente; y siendo además dicha prohibición discriminatoria pues permite un tratamiento diferente frente a aquellos servidores que fueron destituidos y quienes, en cambio, fueron cesados por excedencia, los cuales nunca podrán ingresar a laborar en la Administración Pública, situación incluso más grave que la de aquellos que fueron cesados por haber incurrido en falta grave $[\ldots]{ }^{21}$.

En este pronunciamiento se puede advertir la mera cita o referencia a la idea de proporcionalidad y la falta de un desarrollo, aunque sea mínimo, de su contenido y fundamento normativo ${ }^{22}$. Años después, y ya vigente la Ley del Procedimiento Administrativo General, el Tribunal volverá a

19 Es decir, una proposición para comprender el significado de una expresión, que carece de algún fundamento expuesto con claridad y certeza o de uno que haya establecido la forma en que se puede llegar a alcanzar dicha comprensión. Así, se ha dicho que, a través del intuicionismo, los conceptos y proposiciones: «[...] no pueden ser reconocidas por los cinco sentidos, sino por alguna otra facultad. En algunos autores esta otra facultad es algo así como un sexto sentido; en otros, algo así como la capacidad de intelección apriorística; y en otros, una mezcla de ambas [...] Según todas las teorías que se pueden llamar intuicionistas, la tarea del discurso práctico se resolvería mediante evidencias de algún tipo. Mientras estas evidencias sean suficientes, no queda ningún campo para los argumentos» (ALEXY, Robert. Teoría de la argumentación jurídica. La teoría del discurso racional como teoría de la fundamentación jurídica. Traducción de Manuel Atienza e Isabel Espejo. Prólogo de Manuel Atienza. Lima: Palestra Editores, 2007, pp. 71-72).

20 En sentido estricto, no estamos ante un supuesto de análisis de la potestad sancionadora de la administración en tanto que la prohibición de reingreso a la función pública aparece desligada de una conducta infractora calificada previamente como tal. Sin embargo, el Tribunal hace una analogía respecto de los supuestos de sanciones disciplinarias previstas en el reglamento de la Ley de Bases de la Carrera Pública, y porque, en la práctica, los decretos leyes que establecieron los procedimientos de cese de personal por razón de excedencia constituían sanciones originadas por no acogerse al «retiro voluntario» por incentivos, programa estatal llevado a cabo a partir del año 1992. 21 Tribunal Constitucional: expediente 865-1996-AA/TC.

22 En el mismo sentido, la sentencia recaía en el expediente 903-2003-AA/TC, en donde afirma el carácter desproporcionado de una multa administrativa sin realizar ningún tipo de evaluación o juicio. 
referirse nominalmente al principio de proporcionalidad —y también al de razonabilidad - sin mayores desarrollos ${ }^{23}$.

Recién en el año 2004 podremos encontrar una referencia específica y un desarrollo propio acerca del principio de razonabilidad y de proporcionalidad respecto del ejercicio de las potestades discrecionales de la administración ${ }^{24}$. Ahora bien, en esa oportunidad el Tribunal afirmó que: «[...] más allá de la convención doctrinaria que admite su autonomía como concepto, en puridad, la proporcionalidad es una modalidad más de la razonabilidad (razonabilidad instrumental) $»^{25}$. Con ello daba a entender la inexistencia de un contenido propio del concepto de proporcionalidad distinto al de razonabilidad, y pretendía definirlo señalando que: «[...] exige la existencia indubitable de una conexión directa, indirecta y relacional entre causa y efecto; vale decir, que la consecuencia jurídica establecida sea unívocamente previsible y justificable a partir del hecho ocasionante del acto estatal. En consecuencia, la proporcionalidad lo será cuando la razón del efecto sea deducible de su causa, o previsible a partir de ella» ${ }^{26}$.

Esta sorprendente formulación teórica acerca del contenido del principio de proporcionalidad, sin embargo, será rápidamente superada y dejada de lado, dado que el Tribunal Constitucional acogería una postura mucho más ortodoxa y menos innovadora, como veremos más adelante $^{27}$.

Si seguimos avanzando en el esfuerzo por determinar un contenido propio para el principio de proporcionalidad en el ámbito de las sanciones administrativas, nos encontraremos con que el Tribunal Constitucional establece sus alcances a través de una estrategia que empieza disminuyendo la relevancia de las diferencias entre razonabilidad y proporcionalidad, por un lado, y determinando su fundamento normativo tanto en la cláusula del Estado social y democrático de derecho como en el último párrafo del artículo 200 de la Constitución,

23 «La aplicación de una sanción administrativa constituye la manifestación del ejercicio de la potestad sancionatoria de la Administración. Como toda potestad, está condicionada, en cuanto a su propia validez, a que se respete la Constitución, los principios constitucionales y, en particular, los derechos fundamentales. Al respecto, debe resaltarse que la Administración, en la prosecución de procedimientos administrativos disciplinarios, está obligada ineludiblemente al irrestricto respeto del derecho al debido proceso y, en consecuencia, a los derechos fundamentales procesales y los principios constitucionales, como los de legalidad, razonabilidad, proporcionalidad y defensa, entre otros, que lo conforman» (Tribunal Constitucional: expediente 1199-2003-AA/TC).

24 En la medida en que las sanciones administrativas contemplan el ejercicio de potestades discrecionales, al menos en lo que respecta a la determinación de la sanción aplicable — pues suele ser habitual que la sanción contemple un rango entre un mínimo y un máximo posible que deberá ser fijado en función a los criterios atenuantes o agravantes que la ley determine-, resultará de aplicación la doctrina constitucional sobre el ejercicio de potestades discrecionales. Véase: TORNOS, Joaquín. «Infracción y sanción administrativa: el tema de su proporcionalidad en la jurisprudencia contencioso-administrativa». Revista Española de Derecho Administrativo, número 7, 1975. pp. 607-624, Madrid.

25 Tribunal Constitucional: expediente 090-2004-AA/TC.

26 Tribunal Constitucional: expediente 090-2004-AA/TC.

27 Tribunal Constitucional: expediente 045-2004-AI/TC. 
por el otro ${ }^{28}$. Inmediatamente después, el Tribunal señaló que aunque ha utilizado el principio de proporcionalidad en diversas oportunidades - y con finalidades diversas - no lo había hecho respecto del ejercicio de la potestad sancionadora de la administración ${ }^{29}$, para luego plantear la aplicación —en dicho ámbito- del principio de proporcionalidad, el cual es formulado inicialmente según su expresión clásica -nos referimos al triple juicio de adecuación, necesidad y proporcionalidad en sentido estricto-.

Ahora bien, al momento de aplicar este principio, el Tribunal señaló una matización en la forma de su aplicación específica en el caso del control de las sanciones administrativas, debido a la necesaria atención que la ley aplicable exige de los antecedentes personales del supuesto infractor. Se señala sobre el tribunal que:

[...] esto implica un claro mandato a la administración [...] para que, en el momento de establecer una sanción administrativa, no se limite a realizar un razonamiento mecánico de aplicación de normas, sino que, además, efectúe una apreciación razonable de los hechos en relación con quien los hubiese cometido; es decir, que no se trata solo de contemplar los hechos en abstracto, sino «en cada caso» [...] Por tanto, una decisión razonable en estos casos supone, cuando menos: a) La elección adecuada de las normas aplicables al caso y su correcta interpretación, tomando en cuenta no solo una ley particular, sino el ordenamiento jurídico en su conjunto. b) La comprensión objetiva y razonable de los hechos que rodean al caso, que implica no solo una contemplación en «abstracto» de los hechos, sino su observación en directa relación con sus protagonistas, pues solo así, un «hecho» resultará menos o más tolerable [...] c) Una vez establecida la necesidad de la medida de sanción, porque así lo ordena la ley correctamente interpretada en relación a los hechos del caso que han sido conocidos y valorados en su integridad, entonces el tercer elemento a tener en cuenta es que la

28 «Si bien la doctrina suele hacer distinciones entre el principio de proporcionalidad y el principio de razonabilidad, como estrategias para resolver conflictos de principios constitucionales y orientar al juzgador hacia una decisión que no sea arbitraria sino justa; puede establecerse, prima facie, una similitud entre ambos principios, en la medida en que una decisión que se adopta en el marco de convergencia de dos principios constitucionales, cuando no respeta el principio de proporcionalidad, no será razonable. En este sentido, el principio de razonabilidad parece sugerir una valoración respecto del resultado del razonamiento del juzgador expresado en su decisión, mientras que el procedimiento para llegar a este resultado sería la aplicación del principio de proporcionalidad con sus tres subprincipios: de adecuación, de necesidad y de proporcionalidad en sentido estricto o ponderación" (Tribunal Constitucional: expediente 2192-2004-AA/TC).

29 «El principio de proporcionalidad ha sido invocado en más de una ocasión por este Tribunal, ya sea para establecer la legitimidad de los fines de actuación del legislador en relación con los objetivos propuestos por una determinada norma cuya constitucionalidad se impugna [...] ya sea para establecer la idoneidad y necesidad de medidas implementadas por el Poder Ejecutivo a través de un Decreto de Urgencia [...] o también con ocasión de la restricción de derechos fundamentales en el marco del proceso penal [...] No obstante, este Colegiado no ha tenido ocasión de desarrollar este principio aplicándolo al control de la potestad sancionadora de la Administración, ámbito donde precisamente surgió, como control de las potestades discrecionales de la Administración" (Tribunal Constitucional: expediente 2192-2004-AA/TC). 
medida adoptada sea la más idónea y de menor afectación posible a los derechos de los implicados en el caso ${ }^{30}$.

Como puede apreciarse en la sentencia, el Tribunal Constitucional afirma, en primer lugar, la necesaria aplicación del principio de proporcionalidad para el control de las sanciones administrativas. En segundo lugar, define el principio de proporcionalidad según su fórmula tradicional de un triple juicio de idoneidad, necesidad y proporcionalidad en sentido estricto. En tercer lugar, matiza el contenido anterior afirmando - $\mathrm{O}$, mejor dicho, reconstruyendo- el principio de proporcionalidad para su aplicación en el campo específico de las sanciones administrativas en la forma citada anteriormente. Esta fórmula será repetida por el Tribunal en otras oportunidades ${ }^{31}$.

A nuestro entender, esta reconstrucción del principio de proporcionalidad para ser aplicado específicamente en el ámbito de las sanciones administrativas resulta, por un lado, innecesaria y, por otro, confusa e imprecisa. Resulta innecesaria porque el desarrollo teórico del principio de proporcionalidad es ya lo suficientemente sólido para acoger el principio sin la necesidad de introducir modificaciones o adaptaciones mayores en su contenido ${ }^{32}$. Y resulta confusa e imprecisa porque plantea como criterio interpretativo la realización de un juicio que contenga, como mínimo, tres criterios — que, por cierto, parece recoger los mismos que los tradicionalmente utilizados en el juicio de proporcionalidad-, pero otorgándoles un contenido distinto. Por ejemplo, se refiere a la «elección adecuada» de la norma aplicable, criterio que, ciertamente, constituye un presupuesto lógico de toda tarea interpretativa. En segundo lugar, se refiere a la necesidad de la comprensión objetiva y razonable de los hechos del caso concreto en directa relación con sus protagonistas, todo lo cual es consistente con el concepto de ponderación. Finalmente, plantea como tercer criterio el de la idoneidad de la medida adoptada y su menor afectación posible a derechos fundamentales, con lo que se altera el esquema del principio de proporcionalidad, pues se analiza primero la necesidad de la medida antes que su idoneidad respecto de los fines pretendidos con ella.

Todo lo anterior nos plantea, pues, un esquema interpretativo en el que se busca utilizar los mismos criterios recogidos habitualmente en el principio de proporcionalidad, pero con un contenido distinto, en un orden lógico diferente y planteando, además, que estos criterios son

30 Tribunal Constitucional: expediente 2192-2004-AA/TC.

31 Ver el expediente 3567-2005-AA/TC.

32 Cosa que, por otra parte, ha hecho el propio Tribunal Constitucional al aplicar el principio de proporcionalidad en otras materias, como es el caso de la igualdad de trato. Al respecto, consultar BERNAL PULIDO, Carlos. «La aplicación del principio de proporcionalidad en el juicio de igualdad. Comentario a la sentencia del Tribunal Constitucional del Perú 045-2004-PI/TC». En Miguel Carbonell y Pedro Grández Castro (coordinadores). El principio de proporcionalidad en el Derecho comparado. Lima: Palestra Editores, 2010, pp. 321 y siguientes. 
un listado mínimo a los cuales se podrían añadir otros que no han sido especificados.

A pesar de la pretendida intención ejemplificadora que puede advertirse de esta sentencia, el Tribunal Constitucional dejará de lado los criterios señalados y volverá a un esquema más ortodoxo y sin mayores precisiones respecto de su aplicación en el ámbito de la potestad sancionadora de la administración pública ${ }^{33}$.

\section{A MODO DE CONCLUSIÓN}

De la revisión jurisprudencial que hemos realizado, se puede apreciar que el Tribunal Constitucional ha tenido múltiples oportunidades en las que se ha referido al problema de la proporcionalidad de las sanciones administrativas. En el análisis de los casos estudiados, se puede apreciar que, luego de algunas dudas respecto de la autonomía de los conceptos razonabilidad y proporcionalidad, el tribunal se ha decantado - correctamente, en nuestra opinión - por mantenerse dentro de los alcances del principio de proporcionalidad. Es de destacar, sin embargo, que hubo un momento en que se pretendió establecer un esquema particular de aplicación del principio de proporcionalidad para el caso del ejercicio de la potestad sancionadora de la administración pública pero que, debido a la falta de rigor y coherencia de su formulación, el tribunal rápidamente regresó a una versión mucho más estricta y tradicional —si cabe la expresión - del principio de proporcionalidad, sin que - a la fecha- nuestro supremo intérprete de la Constitución haya construido un esquema interpretativo particular sobre los alcances del principio de proporcionalidad cuando este es utilizado para el control de la potestad sancionadora de la administración pública, tarea que queda pendiente tanto para nuestro Tribunal Constitucional como para nuestra doctrina.

33 «La potestad sancionadora administrativa se orienta bajo los siguientes principios: legalidad, tipicidad, debido procedimiento, razonabilidad, tipicidad, irretroactividad, causalidad, proporcionalidad. El principio de proporcionalidad se encuentra contenido en el artículo $200^{\circ}$ de la Constitución Política (último párrafo) y supone proporción entre los medios utilizados y la finalidad perseguida. Debe existir una correlación entre la infracción cometida y la sanción a aplicar. Se deberá entonces determinar en cada caso concreto si hubo una excesiva afectación de los derechos fundamentales [...] Este principio está estructurado a su vez por tres subprincipios: a) Idoneidad: Toda medida que implique una intervención en los derechos fundamentales debe ser adecuada para contribuir a la obtención de un fin constitucionalmente válido; b) Necesidad: No debe existir otro medio alternativo que, por lo menos, muestre la misma idoneidad para la consecución del fin propuesto y que sea benigno con el derecho afectado, y, c) Proporcionalidad: el grado de intensidad en el que se realice el objetivo de la medida dictada debe ser equivalente al grado de intensidad en el que se afecte el derecho fundamental»" (Tribunal Constitucional: expediente 1767-2007-AA/TC. En el mismo sentido, puede verse la sentencia recaída en el expediente 3951-2007-AA/TC).

\section{PRINCIPIO DE \\ PROPOR- \\ CIONALIDADY \\ SANCIONES \\ ADMINISTRATIVAS \\ EN LA \\ JURISPRUDENCIA \\ CONSTITUCIONAL}

PRINCIPLE OF

PROPORTIONALITY

AND ADMINISTRATI-

VE SANCTIONS ON

CONSTITUTIONAL

CASE LAW 